DOI 10.18699/PlantGen2019-044

\title{
Quantitative real-time PCR as a supplementary tool for molecular cytogenetics
}

\author{
Divashuk M.G. ${ }^{1,2 *}$, Kroupin P.Yu. ${ }^{1,2}$, Nikitina E.A. ${ }^{1}$, Karlov G.I. ${ }^{1,2}$ \\ ${ }^{1}$ Laboratory of Applied Genomics and Crop Breeding, All-Russian Research Institute \\ of Agricultural Biotechnology, Moscow, Russia \\ ${ }^{2}$ Centre for Molecular Biotechnology, Russian State Agrarian University - \\ Moscow Timiryazev Agricultural Academy, Moscow, Russia \\ *e-mail:divashuk@gmail.com
}

Cytogenetics is one of the powerful tools of plant genetics that enables to explore structural and functional organization of the genome as well as evolutionary relationships between species. However, preparing the chromosome slides for analysis and the following procedures such as genomic and fluorescence in situ hybridization (FISH and GISH) are rather expensive, time and labor consuming. Tedious cytogenetics techniques have been highly facilitated by bioinformatic approaches on the basis of whole-genome sequencing data helping not only to reveal new DNA satellite sequences that can be applied as probes for chromosome labelling but also to predict its possible chromosomal localization. Quantitative real-time PCR (qPCR) can be applied in the selection for DNA repeated sequences that can be used as probes for FISH highly efficiently thus increasing the number of successful experiments. qPCR makes it possible to estimate the relative quantity of DNA repeated sequences (satellites or mobile elements) in the genome of the studied species and thus to carry out a preliminary selection of those that can be converted into chromosome- or genome-specific cytogenetic marker. The estimation can be performed even at a semi-quantitative level, therefore, the requirements for primer efficiency are not so strict as for quantitative assessment. We demonstrated the efficiency of qPCR as a supplementary tool for cytogenetics in several case studies, namely, with cytogenetic markers for sex chromosomes of buckthorn, satellite repeats localized to chromosomes of J, St and V genomes in diploid and polyploid grasses (including Thinopyrum intermedium), and tandem repeat-based cytogenetic markers in wheat. New application of qPCR approach has sufficiently reduced the costs and time required for the development of the novel markers that can be widely used in both fundamental and applied research in plant genetics.

Acknowledgements: This research work was supported by Russian Foundation for Basic Research (grant No.17-04-01871a). 